\title{
The Role of Rhodopsin Glycosylation in Protein Folding, Trafficking, and Light-Sensitive Retinal Degeneration
}

\author{
Beatrice M. Tam and Orson L. Moritz \\ Department of Ophthalmology and Visual Sciences and Centre for Macular Research, University of British Columbia, Vancouver, British Columbia, Canada, \\ V5Z 3N9
}

Several mutations in the $\mathrm{N}$ terminus of the G-protein-coupled receptor rhodopsin disrupt NXS/T consensus sequences for N-linked glycosylation (located at N2 and N15) and cause sector retinitis pigmentosa in which the inferior retina preferentially degenerates. Here we examined the role of rhodopsin glycosylation in biosynthesis, trafficking, and retinal degeneration (RD) using transgenic Xenopus laevis expressing glycosylation-defective human rhodopsin mutants. Although mutations T4K and T4N caused RD, N2S and T4V did not, demonstrating that glycosylation at N2 was not required for photoreceptor viability. In contrast, similar mutations eliminating glycosylation at N15 (N15S and T17M) caused rod death. Expression of T17M was more toxic than T4K to transgenic photoreceptors, further suggesting that glycosylation at N15 plays a more important physiological role than glycosylation at N2. Together, these results indicate that the structure of the rhodopsin $\mathrm{N}$ terminus must be maintained by an appropriate amino acid sequence surrounding $\mathrm{N} 2$ and may require a carbohydrate moiety at N15. The mutant rhodopsins were rendered less toxic in their dark inactive states, because RD was abolished or significantly reduced when transgenic tadpoles expressing T4K, T17M, and N2S/N15S were protected from light exposure. Regardless of their effect on rod viability, all of the mutants primarily localized to the outer segment and Golgi and showed little or no endoplasmic reticulum accumulation. Thus, glycosylation was not crucial for rhodopsin biosynthesis or trafficking. Interestingly, expression of similar bovine rhodopsin mutants did not cause rod cell death, possibly attributable to greater stability of bovine rhodopsin.

\section{Introduction}

Mutations affecting glycosylation of the heptahelical G-proteincoupled receptor rhodopsin are associated with retinitis pigmentosa (RP), a disease characterized by progressive degeneration of photoreceptors. Rhodopsin is expressed in rod photoreceptors in the retina and resides in a specialized organelle called the rod outer segment (ROS) in which it is responsible for light capture. The $\mathrm{N}$ terminus of mature rhodopsin is dually glycosylated at residues N2 and N15 (Hargrave, 1977). Mutations of these residues or their surrounding glycosylation consensus sequences (T4K, N15S, and T17M) are linked to a subset of RP known as sector RP in which the inferior retina is primarily affected, potentially attributable to greater light exposure to this region (Fishman et al., 1992; Sullivan et al., 1993; Li et al., 1994; van den Born et al., 1994). Furthermore, both transgenic mice expressing human T17M rhodopsin (White et al., 2007) and a naturally occurring dog model harboring a rhodopsin T4R mutation exhibit lightsensitive retinal degeneration (Kijas et al., 2002; Cideciyan et al., 2005). Together, the human disease and animal models suggest

Received Aug. 28, 2009; revised 0ct. 2, 2009; accepted 0ct. 9, 2009.

This research was funded by grants from the Canadian Institutes of Health Research and the Foundation Fighting Blindness of Canada. O.L.M. is a Canadian Institutes of Health Research New Investigator and W. K. Stell Scholar (Foundation Fighting Blindness (anada). We are grateful to Dr. R. S. Molday for providing mAb $2 B 2$ and Dr. P. Hargrave for providing mAbs B630N and A5-3. We thank Jenny Wong for technical assistance.

Correspondence should be addressed to Orson L. Moritz, Department of Ophthalmology and Visual Sciences, University of British Columbia, 2550 Willow Street, Vancouver, British Columbia, Canada, V5Z 3N9. E-mail: olmoritz@interchange.ubc.ca.

DOI:10.1523/JNEUROSCI.4259-09.2009

Copyright $\odot 2009$ Society for Neuroscience $\quad$ 0270-6474/09/2915145-10\$15.00/0 that glycosylation plays a crucial role in the structure and/or function of rhodopsin.

$\mathrm{N}$-linked glycosylation is the most prevalent posttranslational modification of plasma membrane and secretory proteins and participates in many important biological roles such as protein folding, intracellular targeting, immune response, cell adhesion, and protease resistance. However, a clear role for rhodopsin glycosylation has not been established in vivo. In HEK293S cells, human T17M rhodopsin expresses at low levels, accumulates in the endoplasmic reticulum (ER), and does not appreciably regenerate with its chromophore, 11-cis-retinal (Sung et al., 1991). In COS cells, bovine T4K and T17M rhodopsins only partially regenerate with chromophore (Kaushal and Khorana, 1994). Both studies suggest that defects in rhodopsin glycosylation are associated with impairment of protein folding or stability. However, treatment of COS cells expressing wild-type (WT) bovine opsin with tunicamycin resulted in correctly folded nonglycosylated rhodopsin, suggesting that glycosylation was not crucial for protein folding (Kaushal et al., 1994). Rather, nonglycosylated rhodopsin exhibited reduced ability to activate transducin, which was specifically associated with loss of glycosylation at N15. Further studies suggest that rhodopsin requires glycosylation for incorporation into ROS membranes. In isolated frog retinas treated with tunicamycin, rods synthesized and transported nonglycosylated rhodopsin to the basal ROS at a rate similar to untreated controls. However, nonglycosylated rhodopsin was unable to incorporate normally into disk membranes and was instead released in vesicles into the extracellular matrix (Fliesler and Basinger, 1985; Fliesler et al., 1985). 
Understanding the role of glycosylation on the structure/ function of rhodopsin will facilitate the development of effective therapeutic interventions for rhodopsin-based RP. In this study, we generated transgenic Xenopus laevis expressing single and double mutations that abolish one or both of the glycosylation sites of rhodopsin. We examined the effect of the mutations on expression levels, subcellular localization, and light sensitivity of retinal degeneration (RD). Furthermore, we have begun to differentiate the effects resulting from amino acid substitutions versus those resulting from loss of glycosylation.

\section{Materials and Methods}

Molecular biology. Transgene expression constructs were based on XOP0.8-eGFP-N1 (Tam et al., 2006) in which the green fluorescent protein cDNA is replaced with wild-type or mutant human rhodopsin cDNAs. The wild-type human rhodopsin and wild-type bovine rhodopsin expression plasmids have been described previously (Tam and Moritz, 2006). Human N2S, T4K, T4N, T4V, and bovine T4R mutations were introduced into oligonucleotides used for PCR amplification of rhodopsin cDNAs. The bovine T17M mutation was introduced using a PCR mutagenesis protocol (Nelson and Long, 1989). Human N15S and T17M mutations were introduced using the Quikchange Mutagenesis kit (Stratagene). Introduction of the mutations into cDNAs was verified by DNA sequencing. Expression vectors were linearized by digestion with FseI (New England Biolabs) and purified using the QIAquick Gel Extraction kit (Qiagen) for integration into sperm nuclei.

Generation and rearing of transgenic X. laevis. Transgenic X. laevis tadpoles were generated by the methods described previously (Kroll and Amaya, 1996; Tam and Moritz, 2006, 2007). Embryos were housed in $4 \mathrm{~L}$ tanks in an $18^{\circ} \mathrm{C}$ incubator on a $12 \mathrm{~h}$ light/dark cycle. The light intensity inside the incubator was 1700 lux. Approximately $24 \mathrm{~h}$ after fertilization, embryos were exposed to $20 \mu \mathrm{g} / \mathrm{ml} \mathrm{G} 418$ for $4-5 \mathrm{~d}$ to select for transgenic tadpoles (Moritz et al., 2002). Dark-reared animals were housed in the same incubator as cyclic-reared animals, in opaque tanks wrapped in several layers of aluminum foil. At $14 \mathrm{~d}$ postfertilization (dpf), corresponding to developmental stage 48 , normally developed $X$. laevis were killed and one eye was fixed in $4 \%$ paraformaldehyde buffered with $0.1 \mathrm{M}$ sodium phosphate, $\mathrm{pH} 7.4$, whereas the contralateral eye was solubilized in $100 \mu \mathrm{l}$ of a 1:1 mixture of PBS and SDS-PAGE loading buffer containing $1 \mathrm{~mm}$ EDTA and $100 \mu \mathrm{g} / \mathrm{ml}$ PMSF as described previously (Tam et al., 2006).

Deglycosylation of rhodopsin. Solubilized eye extracts (11 $\mu \mathrm{l})$ were treated with $100 \mathrm{U}$ of PNGaseF (New England Biolabs) in the buffer of the manufacturer supplemented with $1 \% \mathrm{NP}-40$ detergent in a total volume of $15 \mu \mathrm{l}$. Samples were incubated for $1 \mathrm{~h}$ at $37^{\circ} \mathrm{C}$ and then immediately electrophoresed.

Dot blot and Western blot analysis. Dot blots and Western blots of $X$. laevis eye extracts were performed as described previously (Tam et al., 2006). Dot blots were probed with primary monoclonal antibody ( $\mathrm{mAb}$ ) B630N (Adamus et al., 1991) at 1:10 dilution of tissue culture supernatant or mAb 1D4 (MacKenzie et al., 1984) (University of British Columbia, University-Industry Liaison Office) at 1:750 dilution of $1 \mathrm{mg} / \mathrm{ml}$ solution, followed by IR-dye800-conjugated goat anti-mouse antibody at $1: 10,000$ of $1 \mathrm{mg} / \mathrm{ml}$ solution (Rockland). Blots were imaged and quantified using the LICOR Odyssey imaging system. Each dot blot included standards containing $100 \%$ X. laevis rhodopsin (from wild-type retinas) and $100 \%$ transgenic rhodopsin (from transfected cultured cells). The relative affinities of $\mathrm{mAb} B 630 \mathrm{~N}$ (recognizes total rhodopsin) and $\mathrm{mAb}$ 1D4 label (recognizes transgenic rhodopsins) were determined from the control samples and used to calculate the percentage transgenic rhodopsin expression levels. Standard curves derived from serial dilutions of control samples were used to determine the linear range of both antibodies. Plots of expression level versus total rhodopsin were fit to doseresponse curves using SigmaPlot (Systat Software). Nonparametric statistical methods were used for data analysis attributable to nonnormal (i.e., skewed and bimodal) data distributions.

For Western blots, solubilized retinal samples were electrophoresed on $10 \%$ polyacrylamide gels and transferred to a membrane using a semidry blotting apparatus (Bio-Rad). Blots were probed with mAb 1D4 and IR-dye800 secondary antibody and imaged as described for dot blots.

Immunohistochemistry and confocal microscopy. Fixed eyes were embedded and cryosectioned as described previously (Tam et al., 2006). Frozen sections were labeled with mAb 2B2 (Hicks and Molday, 1986) (cell culture supernatant) at 1:10 dilution, followed by 1:750 dilution of cyanine 3-conjugated secondary antibody (Jackson ImmunoResearch) and counterstained with Alexa-488-conjugated wheat germ agglutinin (Invitrogen) and Hoechst 33342 (Sigma-Aldrich) as described previously (Moritz et al., 1999). mAb 2B2 recognizes the $\mathrm{N}$ terminus of rhodopsin. Sections were imaged using a Zeiss 510 laser scanning confocal microscope and $10 \times$ (air) or $40 \times$ (water-immersion) objectives. Postmicroscopy image processing was performed using Adobe Photoshop (Adobe Systems). The brightness and contrast of fluorescent signals derived from antibody labeling were adjusted linearly (i.e., no changes to gamma settings) to preserve the relative intensities from different parts of the cell. However, signals derived from wheat germ agglutinin and Hoechst 33342 staining were adjusted in a nonlinear manner to best represent the architecture of the retina. All adjustments to brightness and contrast were done to entire images before cropping.

\section{Results}

\section{Human but not bovine glycosylation-deficient rhodopsin mutants cause retinal degeneration in transgenic $X$. laevis} Mutations that interfere with the N-linked glycosylation of rhodopsin cause RP in humans and light-sensitive RD in animal models. To study the mechanisms underlying rod death associated with these mutations, we generated primary transgenic $X$. laevis expressing human WT, T4K, or T17M rhodopsins $(n=29$ per group) under the control of the $X$. laevis rod opsin promoter. At $14 \mathrm{dpf}$, one eye was solubilized for quantitative dot blot analysis, whereas the contralateral eye was fixed for immunohistology. Dot blots (Fig. $1 A$ ) probed with either mAb B6-30N (recognizes both transgenic and endogenous rhodopsin) or mAb 1D4 (recognizes transgenic rhodopsin only) were used to determine total and transgenic rhodopsin (as a percentage of total rhodopsin) levels. Consistent with our previously published work, a wide range of expression levels was observed for each transgene attributable to independent integration sites and variable copy number (Fig. 1 B) (Tam et al., 2006). Also as reported previously, regardless of the human WT rhodopsin expression levels obtained in our experiments, total rhodopsin levels remained constant, demonstrating that human WT rhodopsin is not toxic to X. laevis photoreceptors (Tam and Moritz, 2007). In contrast, expression of human T4K and T17M rhodopsins was associated with decreased total rhodopsin, indicating that these two mutants were toxic and caused RD. On average, retinas expressing hT4K rhodopsin and hT17M rhodopsin contained 29 and $62 \%$ less total rhodopsin than those expressing hWT rhodopsin, respectively $[p<0.0005$, Kruskal-Wallis test, followed by multiple comparisons according to Conover (1999)]. Cryosections of retinas from contralateral eyes were also examined (Fig. 1C). Retinas expressing human WT rhodopsin appeared healthy with numerous rods and long, densely packed ROS. In contrast, many retinas expressing human T4K or T17M rhodopsins showed central regions of absent or shortened rods.

In a similar experiment, we compared transgenic $X$. laevis expressing bovine WT, T4R, and T17M rhodopsin $(n=29$ per group). As described previously (Tam and Moritz, 2007), expression of bovine wild-type rhodopsin had no deleterious effects on $X$. laevis rods. Interestingly, the mutant bovine rhodopsins also did not cause RD as determined by dot blot (Fig. $2 \mathrm{~A}$ ) and histological analysis (Fig. $2 \mathrm{~B}$ ). Altogether, these results indicate that $\mathrm{RP}$ induced by glycosylation-deficient mutants can be modeled 
A
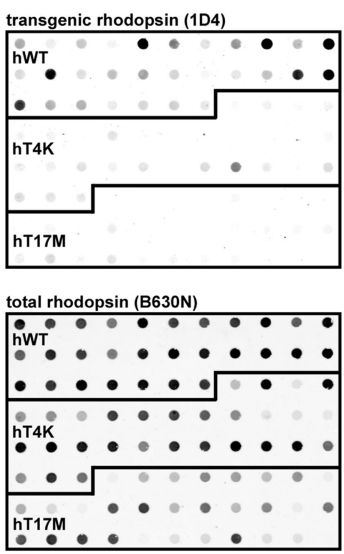

C

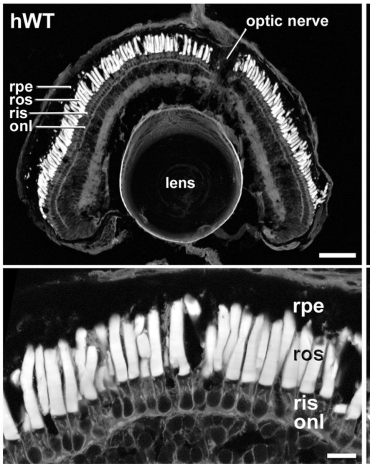

B
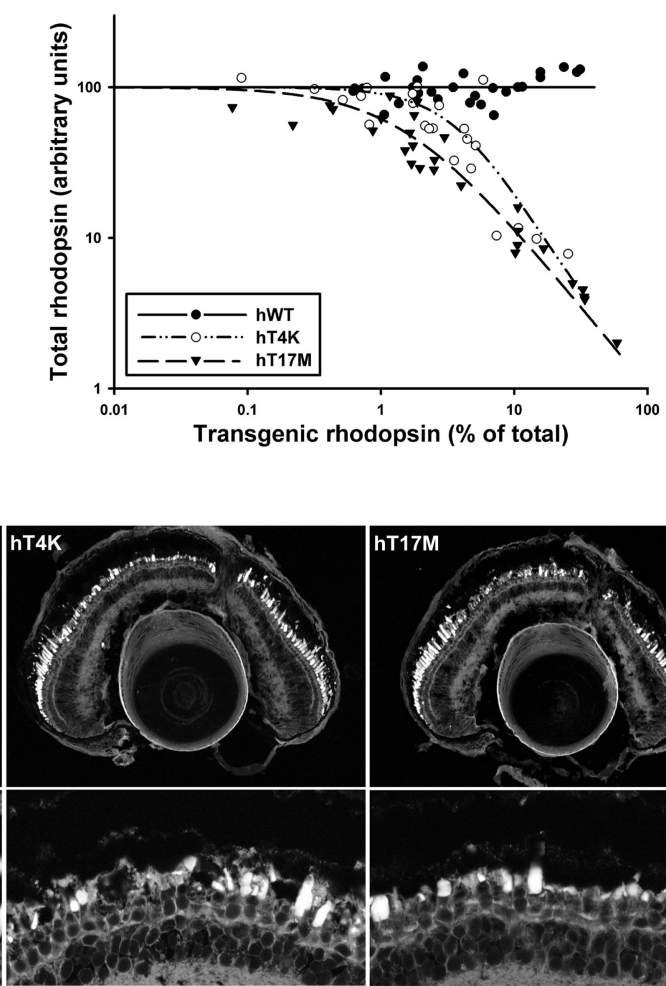

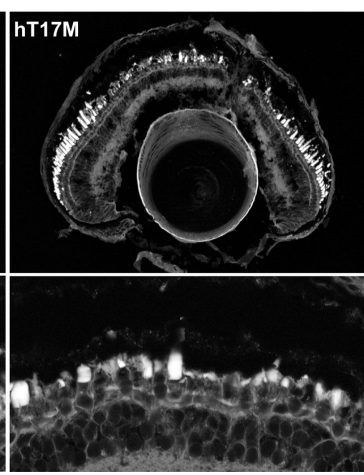

Figure 1. Expression of human T4K and hT17M rhodopsin causes RD in transgenic $X$. laevis rod photoreceptors. $A$, Solubilized retinal extracts from primary transgenic tadpoles ( $n=29$ per group) expressing human WT, T4K, or T17M rhodopsin were spotted on membranes and probed with mAb B630N (recognizes total rhodopsin) or mAb $1 D 4$ (recognizes only transgenic rhodopsin). Varying levels of transgenic rhodopsin signals were obtained within and between the different groups. $\boldsymbol{B}$, Fluorescent signals from dot blots were quantified and used to derive plots of transgenic rhodopsin expression levels versus total rhodopsin levels. Expression of hWT rhodopsin did not affect total rhodopsin levels, whereas increasing expression of both hT4K and hT17M rhodopsin correlated with a loss of total rhodopsin, which is indicative of RD. C, Confocal micrographs of cryosections from transgenic retinas stained with wheat germ agglutinin. Top row shows the entire eye, and bottom row shows higher magnification of the centra retina. Retinas expressing hWT rhodopsin (left) appeared healthy with long closely packed ROS. In contrast, retinas expressing hT4K (middle) and hT17M (right) rhodopsin exhibited retinal degeneration as seen by loss or shortening of ROS. rpe, Retinal pigment epithelium; ros, rod outer segments; ris, rod inner segments; onl, outer nuclear layer. Scale bars: top row, $100 \mu \mathrm{m}$; bottom row $20 \mu \mathrm{m}$.

\section{A}

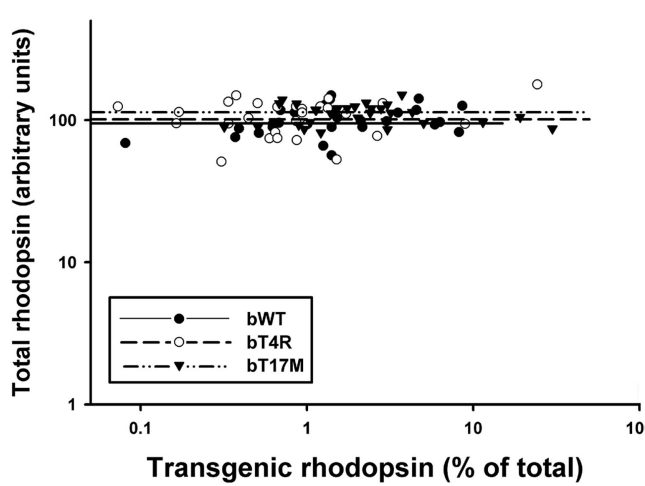

B

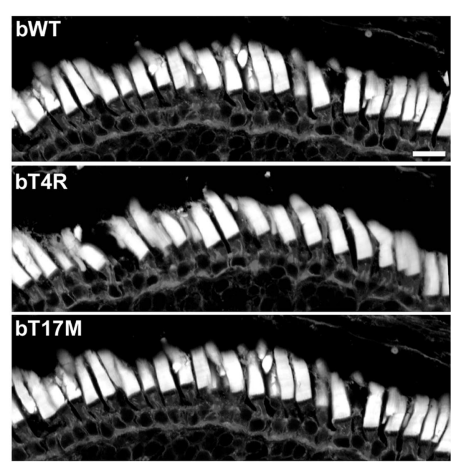

Figure 2. Expression of bovine T4R and T17M rhodopsins does not cause retinal degeneration in transgenic $X$. laevis rod photoreceptors. $A$, Plot of transgenic rhodopsin versus total rhodopsin derived from dot blots of solubilized retinal extracts from primary transgenic tadpoles ( $n=29$ per group) expressing bovine WT, T4R, and T17M rhodopsin. No reduction in total rhodopsin was observed in any of the groups regardless of expression level or transgene. $\boldsymbol{B}$, Confocal micrographs of cryosections of transgenic retinas expressing bWT (top), bT4R (middle), and bT17M (bottom) stained with wheat germ agglutinin. Retinal degeneration was not observed regardless of which transgene was expressed; bT4R and bT17M expressing retinas were similar to bWT expressing retinas with respect to ROS length and density. Scale bar, $20 \mu \mathrm{m}$.

using human rhodopsin mutants in transgenic $X$. laevis but not using bovine rhodopsin mutants.

Dark rearing of transgenic tadpoles expressing human $\mathrm{T} 4 \mathrm{~K}$ and T17M rhodopsins prevents retinal degeneration

In humans, RP caused by $\mathrm{T} 4 \mathrm{~K}$ and $\mathrm{T} 17 \mathrm{M}$ assumes a sector phenotype in which the inferior retina is preferentially affected, possibly attributable to the greater light exposure of the lower retina from overhead light sources such as the sun or indoor ceiling lighting. To determine whether our X. laevis models exhibit light-sensitive $\mathrm{RD}$, we raised primary transgenic tadpoles ( $n=40$ per group) in either $12 \mathrm{~h}$ light/ dark cycle or constant darkness and compared the extent of RD between the two conditions. As seen in the initial experiment, when tadpoles expressing human $\mathrm{T} 4 \mathrm{~K}$ rhodopsin were raised in cyclic light, total rhodopsin levels decreased with increasing transgenic rhodopsin levels (Fig. $3 A$ ) and loss of ROS was observed in retinal cryosections (Fig. 3B, bottom). However, when tadpoles were raised in constant darkness, no loss of total rhodopsin was observed even at higher transgene expression levels (Fig. 3A) and retinal histology appeared normal (Fig. $3 B$, top). Almost identical results were obtained for tadpoles expressing human T17M rhodopsin (Fig. 3C,D). These results indicate that T4K- and T17Minduced RDs were associated with light exposure and protection from lightrescued rods from cell death.

\section{Human T4K and T17M rhodopsin} express at wild-type levels and localize predominantly to the ROS in transgenic photoreceptors

In cultured human kidney cells, human T17M rhodopsin is expressed at low levels and accumulates in the ER (Sung et al., 1991). In monkey kidney cells, bovine T4K and T17M rhodopsins are expressed at high levels and present in mature form on Western blots but only partially reconstitute with 11-cis-retinal (Kaushal and Khorana, 1994). These results suggest substantial (human mutants) or subtle (bovine mutants) defects in the folding of these glycosylation mutants in in vitro systems. To assess the capacity for these glycosylation mutants to fold and traffic properly in vivo, we determined their expression levels and subcellular localization in transgenic $X$. laevis retinas.

Primary transgenic tadpoles expressing human WT, T4K, and T17M rhodop- 
$\sin (n=29$ per group) were raised in the dark. We determined transgenic rhodopsin levels from dot blot analysis of the solubilized eyes (Fig. 4A). There was no statistical difference between hWT, hT4K, and hT17M rhodopsin expression levels ( $p=0.285$, Kruskal-Wallis test). Therefore, under dark-rearing conditions, hT4K and hT17M did not appear to be prone to defects in biosynthesis such as misfolding or degradation.

Retinal cryosections of transgenic tadpoles raised in cyclic light or constant dark were immunolabeled with $\mathrm{mAb} 2 \mathrm{~B} 2$, which only recognizes mammalian (transgenic) rhodopsins (Fig. 4B). As expected, human WT rhodopsin localized primarily to the ROS and Golgi membranes. Little or no labeling was seen in the ER, inner segment plasma membrane, or synapse. Anti-rhodopsin immunolabeling of $X$. laevis retinas typically exhibits a "halo" distribution around the periphery of the ROS attributable to high concentrations of the antibody epitope in tightly packed disk membranes, resulting in poor antibody penetration of the interior of the ROS (Luo et al., 2004; Tam and Moritz, 2006). Regardless of the light-rearing conditions, both T4K and T17M rhodopsins resembled WT rhodopsin in their distribution, suggesting that even when subjected to light exposure, these mutants did not accumulate in the ER and thus were not significantly impaired with respect to protein folding. Similar results were obtained for the localization of bovine T4R and $\mathrm{T} 17 \mathrm{M}$ rhodopsin (data not shown). In contrast, human $\mathrm{P} 23 \mathrm{H}$ rhodopsin (a well characterized misfolding mutant) localizes predominantly to the photoreceptor ER (Tam and Moritz, 2007; this study).

\section{Comparison of human $\mathrm{T} 4 \mathrm{~K}$ and $\mathrm{T} 17 \mathrm{M}$ rhodopsins reveals differences in glycosylation and cell toxicity}

Studies of T4K and T17M mutants expressed in cultured cells suggest that the two glycans of rhodopsin may fulfill distinct functional roles (Kaushal et al., 1994). Furthermore, analysis of frog and human rhodopsins suggests that glycans at N2 might be structurally different from those at N15 (Duffin et al., 1993; Fujita et al., 1994). We therefore directly compared the two mutants with respect to their effects on $\mathrm{RD}$ and their glycosylation state.

Total rhodopsin levels of transgenic tadpole retinas reared in cyclic light were obtained from the dot blots used to derive the dose-response curves in Figure 3. As a group, retinas expressing $\mathrm{T} 17 \mathrm{M}$ contained significantly less total rhodopsin than those expressing T4K (Fig. $5 A$, average values are 47.5 vs 63.2 arbitrary units, $p=0.026$, Mann-Whitney test). Moreover, the shift in the dose-response curve (Fig. $5 A$ ) indicates that T17M was capable of inducing $\mathrm{RD}$ at lower expression levels than T4K. A similar shift is seen in Figure $1 B$. Thus, human T17M rhodopsin was more toxic to rods than $\mathrm{T} 4 \mathrm{~K}$ rhodopsin.

The glycosylation states of the two mutants were compared by treatment of retinal extracts with or without PNGaseF, followed by SDS-PAGE and Western blotting. PNGaseF cleaves N-linked
B

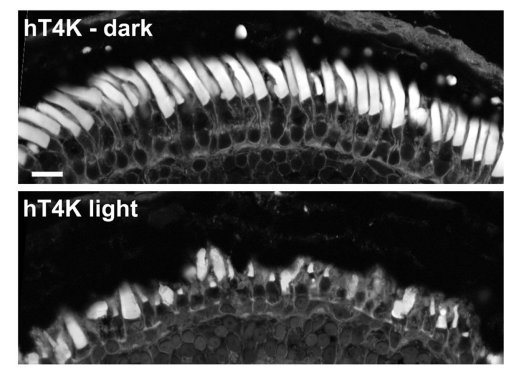

D

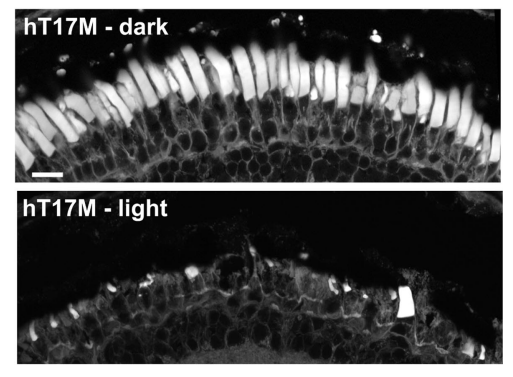

Figure 3. Dark rearing rescues retinal degeneration caused by expression of hT4K and hT17M rhodopsin. Transgenic tadpoles extracts were dot blotted and subjected to Western blot analysis ( $n=40$ per group). A, C Fluorescent signals were ofs of total rhodopsin was observed when tadpoles were reared in the dark. $\boldsymbol{B}, \boldsymbol{D}$, Confocal micrographs of cryosections of (bottom). However, retinas of animals reared in constant dark retained long closely packed ROS similar to those of

oligosaccharide from glycoproteins. mAb 1D4 was used to probe Western blots for transgenic rhodopsins (Fig. 5C). As predicted, both human T4K and T17M rhodopsins migrated faster than human WT rhodopsin, attributable to lack of one of the two glycans. However, human T4K also migrated faster than T17M, suggesting that the two carbohydrate moieties are not structurally identical. After treatment with PNGaseF, all transgenic rhodopsins migrated at the same rate, confirming that the differences in mobility between the mutants are attributable to differences in glycosylation rather than the amino acid substitutions.

Interestingly, a similar analysis of bovine T4R and T17M rhodopsins (Fig. 5D) demonstrated that, unlike the human mutants, each bovine mutant adopts two distinct glycoforms. The mobilities of all four species observed were intermediate between fully glycosylated and fully deglycosylated rhodopsin, and therefore each band probably represents a unique monoglycosylated form.

Collectively, these results suggest that the oligosaccharides at positions N2 and N15 of human rhodopsin are neither structurally identical nor equivalent in their functional importance to rhodopsin.

\section{Glycosylation is not required for rhodopsin biosynthesis,} targeting, or insertion into disk membranes

In studies performed on frog retinas, treatment with the glycosylation inhibitor tunicamycin did not affect rhodopsin synthesis and intracellular transport but significantly impaired disk morphogenesis (Fliesler and Basinger, 1985; Fliesler et al., 1985). However, in the present study, both T4K and T17M rhodopsins primarily localized to the ROS, suggesting that a single oligosac- 
A

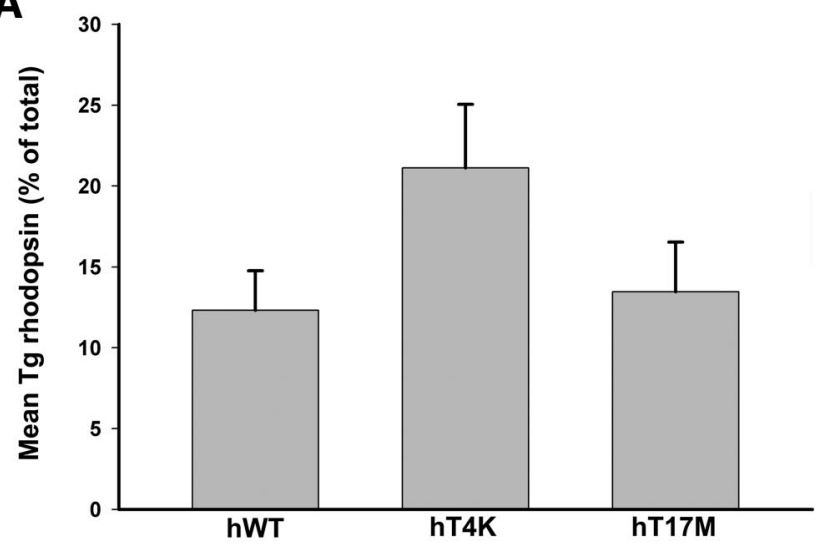

B

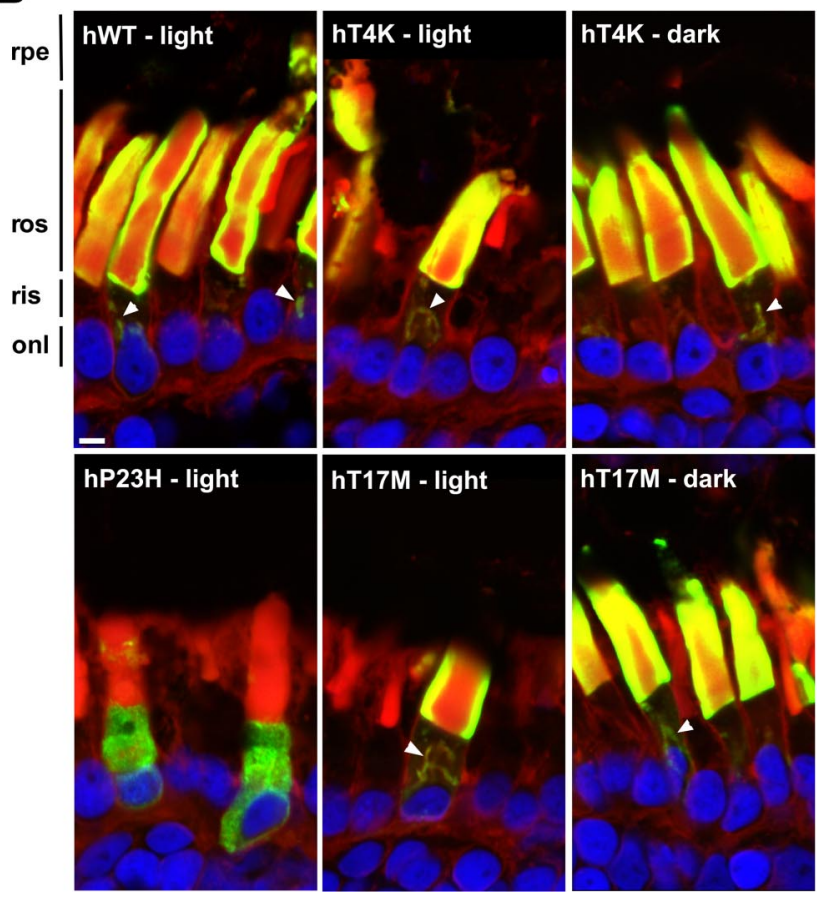

Figure 4. hT4K and hT17M rhodopsin exhibit normal biosynthesis and trafficking. $\boldsymbol{A}$, Transgenic rhodopsin levels were determined from dot blots of retinal extracts from primary transgenic animals expressing either hT4K or hT17M rhodopsin ( $n=29$ per group) and reared in constant dark for $14 \mathrm{~d}$. Mean expression levels of hWT, hT4K, and hT17M were not statistically significant. B, Confocal micrographs of retinal cryosections labeled with mAb 2B2 (green), which recognizes transgenic rhodopsin only, and counterstained with wheat germ agglutinin (red) and Hoechst nuclear stain (blue). The overlap of green and red signals is represented by yellow. Human WT rhodopsin localized primarily to the ROS and Golgi membranes (arrowheads). The same pattern of distribution was exhibited by hT4K and hT17M rhodopsins regardless of whether tadpoles were raised in cyclic light or constant dark. In contrast, hP23H rhodopsin was retained in the ER of the rod inner segments, a distribution highly suggestive of protein misfolding. Thus, both hT4K and hT17M rhodopsin appear to fold and traffic properly. Fluorescent signals derived from antibody labeling were adjusted linearly, whereas signals derived from wheat germ agglutinin and Hoechst staining were adjusted nonlinearly to better represent the architecture of the retina. rpe, Retinal pigment epithelium; ros, rod outer segments; ris, rod inner segments; onl, outer nuclear layer. Scale bar, $5 \mu \mathrm{m}$.

charide chain is sufficient for incorporation into ROS disk membranes. To further examine the role of glycosylation in rhodopsin trafficking and disk morphogenesis, we constructed a series of three human rhodopsin mutants (N2S, N15S, and N2S/N15S), which eliminate either or both glycosylation sites. Solubilized retinal extracts from $14 \mathrm{dpf}$ primary transgenic tadpoles were analyzed by quantitative dot blot $(n=22$ per group) to generate dose-response curves (Fig. 6A). N2S rhodopsin was expressed at levels comparable with WT, and total rhodopsin content was not significantly different from WT $[p=0.379$, Kruskal-Wallis test, followed by multiple comparisons according to Conover (1999)]. Retinas expressing N2S rhodopsin exhibited long densely packed ROS (Fig. $6 \mathrm{~B}$ ), and the protein localized primarily to ROS and Golgi membranes (Fig. 6C), similar to WT rhodopsin. In contrast, both N15S and N2S/N15S caused significant RD (Fig. $6 A, B)$; total rhodopsin levels decreased by 63 and $82 \%$, respectively, compared with retinas expressing human WT rhodopsin $\left(p<1 \times 10^{-9}\right)$. Similar to N2S rhodopsin, both N15S mutants localized predominantly to the ROS (Fig. 6C). In retinas expressing higher levels of N2S/N15S rhodopsin, a small proportion of the mutant rhodopsin was also observed in the ER of some rods (supplemental Fig. 1, available at www.jneurosci.org as supplemental material), suggesting that the absence of both glycans may cause limited protein misfolding. However, even in rods exhibiting ER localization, the majority of mutant rhodopsin still trafficked to the ROS. Thus, although the lack of glycosylation at N2 versus N15 resulted in very different outcomes with respect to cell viability, neither glycan was required for rhodopsin biosynthesis, trafficking, or insertion into disk membranes.

\section{Structural requirements surrounding N2}

Human N2S rhodopsin did not cause significant RD, indicating that glycosylation at this site was not crucial for photoreceptor survival. However, because T4K rhodopsin did cause rod death, the substitution of lysine (a large charged amino acid) for threonine (a small uncharged amino acid) must be the primary defect, suggesting that the structure of the $\mathrm{N}$ terminus of rhodopsin may be intolerant of amino acid substitutions. To probe the structural requirements of this region, we generated transgenic $X$. laevis expressing human rhodopsin with two other substitutions, T4V and T4N. Like lysine, asparagine is hydrophilic albeit neutral in charge, whereas valine is hydrophobic. Solubilized retinal extracts from $14 \mathrm{dpf}$ primary transgenic tadpoles were analyzed by quantitative dot blot ( $n=22$ per group) to generate doseresponse curves (Fig. 7A). The curve obtained for T4V rhodopsin was similar to WT and N2S rhodopsin (i.e., high expression with no indication of RD), whereas increasing levels of T4N resulted in decreased total rhodopsin. Total rhodopsin levels in retinas expressing T4V rhodopsin were not significantly different from retinas expressing WT rhodopsin $[p=0.146$, Kruskal-Wallis test, followed by multiple comparisons according to Conover (1999)]. In contrast, total rhodopsin levels in retinas expressing T4N rhodopsin were significantly lower than retinas expressing WT rhodopsin $\left(p=3.11 \times 10^{-5}\right)$. Cryosections confirmed lack of RD in T4V-expressing retinas, whereas T4N-expressing retinas exhibited rod loss (Fig. 7B). Both T4N and T4V rhodopsins trafficked predominantly to the ROS with little or no accumulation in the ER (Fig. 7C). Thus, the $\mathrm{N}$ terminus of rhodopsin has specific structural requirements such that not all amino acid substitutions at this position are tolerated by rod photoreceptors. However, glycosylation at N2 is not one of these requirements.

\section{Dark rearing significantly rescues retinal degeneration} induced by hN2S/N15S rhodopsin

Retinal degenerations caused by both monoglycosylated hT4K and hT17M were rescued by dark rearing, suggesting that rods tolerated the absence of a single glycosylation site when rhodopsin was in its inactive state. We therefore sought to determine whether rod photoreceptors of dark-reared tadpoles would also tolerate a completely nonglycosylated form of rhodopsin. Pri- 
mary transgenic tadpoles expressing N2S/ N15S rhodopsin were reared in either cyclic light or constant dark. Solubilized retinal extracts from $14 \mathrm{dpf}$ tadpoles were analyzed by quantitative dot blot $(n=17$ per group) to generate dose-response curves (Fig. 8 A). Much greater total rhodopsin loss occurred in transgenic tadpoles raised in cyclic light compared with those raised in constant darkness. On average, transgenic tadpoles reared in constant dark had 2.3-fold higher total rhodopsin levels ( $p=1.81 \times 10^{-4}$, Mann-Whitney test). Although protection from light exposure did not entirely prevent $\mathrm{RD}$, the effects on retinal histology were subtle (Fig. $8 \mathrm{~B}$ ). The relatively small loss of total rhodopsin in dark-reared animals may be attributable to the slight propensity for this mutant to misfold. Altogether, these results indicate that the inactive form of nonglycosylated rhodopsin was better tolerated by rod photoreceptors than the light-activated form.

\section{Discussion}

The oligosaccharides of glycoproteins influence protein properties such as folding kinetics, quality control, stability, protease resistance, enzymatic activity, and protein-protein interactions. However, little is known about the roles of specific glycans of specific proteins. Rhodopsin contains two N-linked glycosylation consensus sequences, both of which are associated with mutations that cause RP. In this study, we examined the phenotypes associated with loss of either or both of the glycans of rhodopsin and began to distinguish between the effects of amino acid substitutions versus those of lack of glycosylation. We demonstrate that light-sensitive RD can be modeled in transgenic $X$. laevis expressing RP-linked human rhodopsin glycosylation mutants. We show that glycosylation at N2 is not crucial for rhodopsin biosynthesis, trafficking, and disk membrane insertion, or photoreceptor viability. Nonetheless, the surrounding region (minimally encompassing the first four amino acids) contains crucial structural information such that some but not all amino acid substitutions induced RD. In contrast, both mutations affecting glycosylation at N15 were toxic to rods under normal rearing conditions, suggesting a direct role for this carbohydrate moiety in rhodopsin structure/function. Furthermore, RDs caused by mutations in both glycosylation consensus sequences were significantly rescued by dark rearing, suggesting that the structure surrounding N2 and the oligosaccharide at N15 play crucial roles specifically in the active conformation of rhodopsin.

All of the rhodopsin glycosylation mutants investigated in this study localized primarily to the ROS. To our knowledge, this study is the first to determine the in vivo localization of the human RP-causing mutants T4K, N15S, and T17M rhodopsin. Although a mouse model expressing human T17M rhodopsin exists (Li et al., 1998), localization of the mutant rhodopsin was not determined. In a naturally occurring dog model of RP, mutant T4R rhodopsin localizes to the ROS (Zhu et al., 2004). Our results indicate that nonglycosylated rhodopsin (N2S/N15S rhodopsin) was capable of targeting to and inserting into ROS disk membranes. In contrast, in previous studies of tunicamycin-

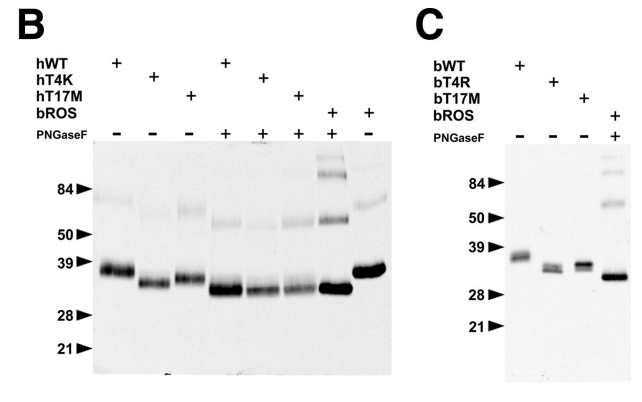

Figure 5. Comparison of hT4K and hT17M with respect to toxicity and glycosylation state. Transgenic animals expressing either and $\mathrm{hT17M}$ expression levels versus total rhodopsin levels. The average total rhodopsin levels are significantly lower in animals

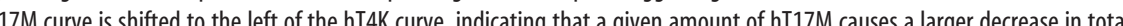
hodopsin than does the same amount of hT4K. $\boldsymbol{B}$. Western blots of transgenic retinas expressing hWT, hT4K, or hT17M rhodopsins probed with mAb 1D4 (recognizes transgenic rhodopsin only). Solubilized retinal extracts were incubated with or without

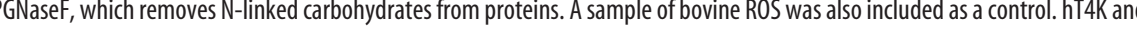

treated frog retinas, nonglycosylated rhodopsin was transported to the apical inner segment (Fliesler et al., 1985) but was unable to incorporate into disk membranes and instead was shed as extracellular vesicles (Fliesler and Basinger, 1985). It is possible that nonglycosylated rhodopsin is incapable of initiating disk morphogenesis but is capable of entering disks formed in the presence of normally glycosylated rhodopsin. Alternatively, because tunicamycin is a nonspecific inhibitor of GlcNAc phosphotransferase, the inability of nonglycosylated rhodopsin to incorporate into disks may have been mediated by other glycoproteins that participate in disk morphogenesis, such as prominin-1 (Maw et al., 2000; Yang et al., 2008). Nonetheless, in our model system, N2S/N15S rhodopsin was expressed on a wild-type rhodopsin background, which accurately reflects autosomal dominant RP in which the patients are heterozygous.

Little or no transgenic mutant rhodopsins accumulated in the ER, suggesting that these mutations did not significantly affect protein folding. However, based on expression in HEK293 cells, human $\mathrm{T} 17 \mathrm{M}$ rhodopsin, like $\mathrm{P} 23 \mathrm{H}$ rhodopsin, expresses at very low levels, accumulates in the ER, and does not reconstitute appreciably with 11-cis-retinal (Sung et al., 1991). A recent study that modeled protein folding in the ER suggests that subtle differences in the concentrations of pathway components involved in ER export and retrotranslocation can have dramatic effects on ER export efficiency, potentially explaining frequently observed misfolding of membrane proteins in heterologous expression systems (Wiseman et al., 2007). Such differences may also explain the apparently contradictory results observed with T17M rhodopsin in photoreceptors versus cultured cells.

It is possible that, in transgenic retinas exposed to cyclic light, rods in which mutant rhodopsin accumulated in the ER died and thus were not visualized. However, one would expect a continuum of ER accumulation, increasing from undetectable to the level at which cell death is initiated. Sublethal amounts of misfolded $\mathrm{P} 23 \mathrm{H}$ rhodopsins can be easily visualized in the ER of transgenic X. laevis rods (Tam and Moritz, 2007). We examined retinas expressing the human glycosylation mutants at varying levels but did not observe a pattern of increasing ER accumula- 
A

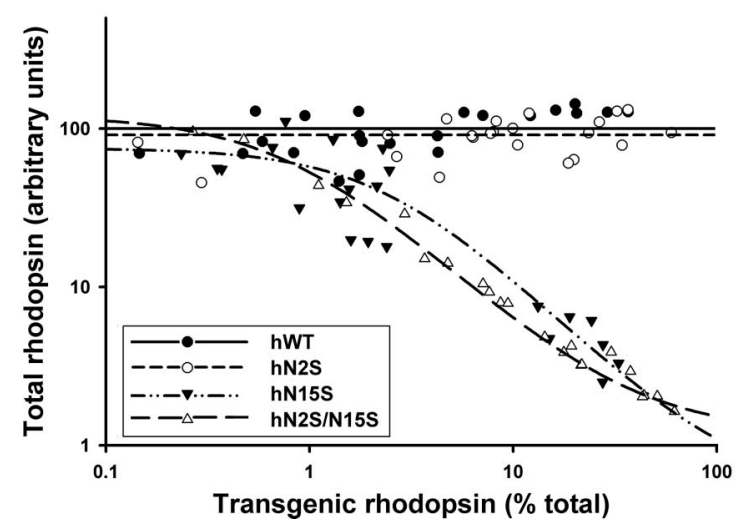

B

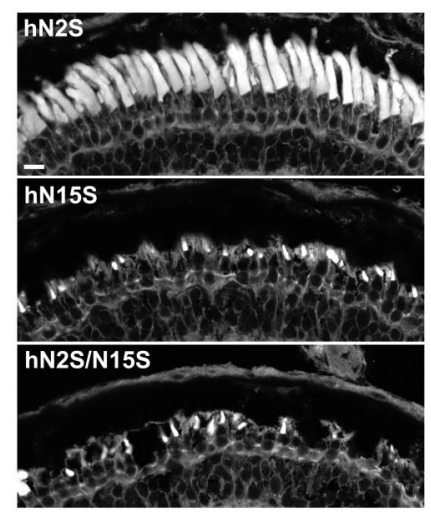

C

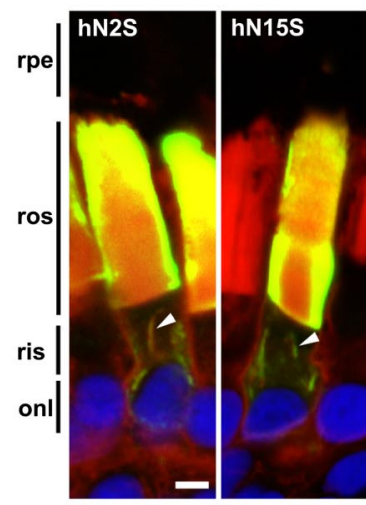

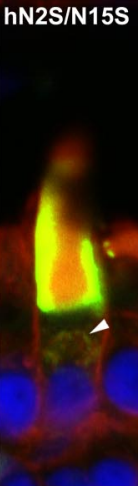

Figure 6. Glycosylation of rhodopsin is not required for biosynthesis or trafficking but may be required at N15 for rod cell viability. Transgenic animals expressing hWT, hN2S, hN15S, or hN2S/N15S ( $n=22$ per group) were generated and raised in cyclic light. Solubilized eyes were analyzed by dot blot and immunohistochemistry. $A$, Plot of transgenic versus total rhodopsin levels. Expression of hWT or hN2S rhodopsin did not affect total rhodopsin levels, whereas expression of hN15S or hN2S/N15S resulted in a decrease of total rhodopsin. $\boldsymbol{B}, \boldsymbol{C}$, Confocal micrographs of cryosections from transgenic retinas expressing hN2S, hN15S, or hN2S/N15S rhodopsins. (ryosections were stained with wheat germ agglutinin (B) or labeled with mAb 2B2 (green; recognizes transgenic rhodopsin only) and counterstained with wheat germ agglutinin (red) and Hoescht nuclear dye (blue) (C). Retinas expressing hN2S appeared healthy with long, densely packed ROS. However, many retinas expressing either hN15S or hN2S/N15S exhibited retinal degeneration characterized by short or absent ROS. Immunolabeled cryosections demonstrate that all three mutants localized primarily to the ROS and Golgi membranes (arrowheads) regardless of whether or not they induced retinal degeneration. Fluorescent signals derived from antibody labeling were adjusted linearly, whereas signals derived from wheat germ agglutinin and Hoechst staining were adjusted nonlinearly to better represent the architecture of the retina. rpe, Retinal pigment epithelium; ros, rod outer segments; ris, rod inner segments; onl, outer nuclear layer. Scale bars: $\boldsymbol{B}$, 20 $\mu \mathrm{m} ; \mathrm{C}, 5 \mu \mathrm{m}$.

A

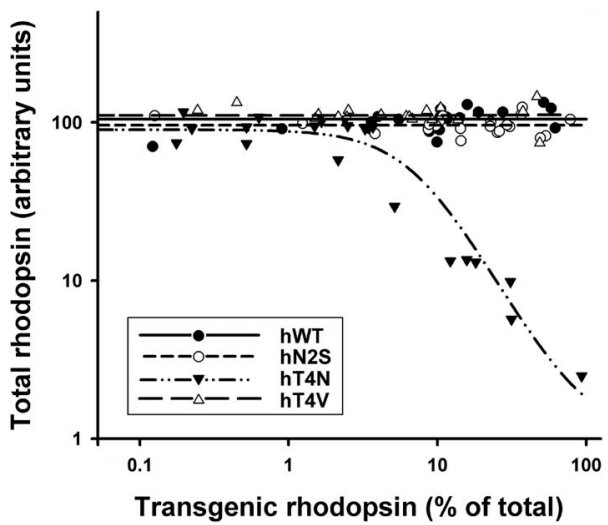

B

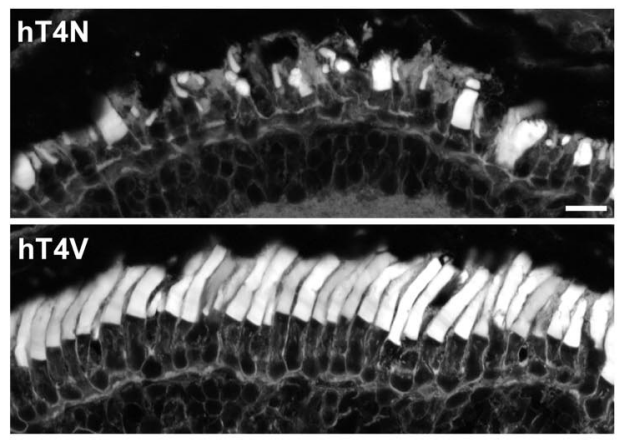

C

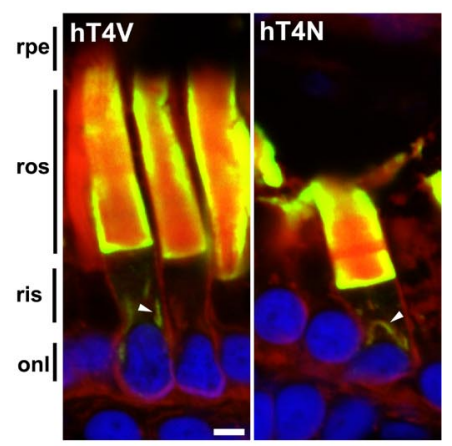

Figure 7. The amino acid sequence of the extreme N terminus rather than glycosylation of N2 is crucial for rod cell viability. Transgenic animals expressing hWT, hN2S, hT4N, or hT4V ( $n=22$ per group) were generated and raised in cyclic light. $\boldsymbol{A}$, Solubilized retinal extracts were analyzed by dot blot to derive plot of transgenic versus total rhodopsin levels. Expression of hWT or hN2S rhodopsin did not affect total rhodopsin levels as shown in Figure 6. Similarly, total rhodopsin levels in retinas expressing hT4V remained constant. However, expression of hT4N resulted in a decrease of total rhodopsin. B, C, Cryosections were stained with wheat germ agglutinin (B) or labeled with mAb 2B2 (green; recognizes transgenic rhodopsin only) and counterstained with wheat germ agglutinin (red) and Hoescht nuclear dye (blue) (C). Retinas expressing hT4V appeared healthy with long, densely packed ROS. However, many retinas expressing hT4N exhibited retinal degeneration characterized by short or absent ROS. In cryosections labeled with mAb 2B2, both mutants localized primarily to the ROS and Golgi membranes (arrowheads). Fluorescent signals derived from antibody labeling were adjusted linearly, whereas signals derived from wheat germ agglutinin and Hoechst staining were adjusted nonlinearly to better represent the architecture of the retina. rpe, Retinal pigment epithelium; ros, rod outer segments; ris, rod inner segments; onl, outer nuclear layer. Scale bars: $B, 20 \mu \mathrm{m} ; \boldsymbol{C}, 5 \mu \mathrm{m}$.

tion, even in rods that exhibited significantly shortened ROS or nuclear condensation. Moreover, RD was most severe in the central retina and less pronounced at the periphery, in which rods are younger or just developing. We did not observe significant differences in the distribution of mutant rhodopsins between cells of the central and peripheral retina.

A defect in primary structure rather than lack of glycosylation at N2 underlies the pathogenic mechanism of T4K rhodopsin. Expression of a transgenic rhodopsin lacking the first glycosylation site (N2S) had no significant effect on rod viability. Moreover, although both T4N and T4K caused RD, T4V did not.
Interestingly, both threonine and valine are $\mathrm{C}-\beta$ branched amino acids, i.e., their C- $\beta$ carbons have two non-hydrogen substituents, whereas most amino acids have one. Because of increased bulkiness near the peptide backbone, these amino acids are preferentially found within $\beta$ sheets rather than $\alpha$ helices or turns (Williams et al., 1987). In contrast, lysine and asparagine residues typically have low preference for $\beta$ sheets. The crystal structure of bovine rhodopsin indicates that the region between Gly3 to Pro12 forms two antiparallel strands in a typical $\beta$-sheet fold (Palczewski et al., 2000). Together with the other extracellular loops of rhodopsin, the $\mathrm{N}$ terminus combines to form a multilay- 
ered structure referred to as the "retinal plug," which lies over top of the chromophore binding pocket (Bourne and Meng, 2000; Filipek et al., 2003). Although the introduction of a positive charge may destabilize this region in the case of T4K, as is proposed for the T4R dog (Zhu et al., 2004), our results obtained from the T4N mutant suggest that the underlying reason is loss of the $\beta$-sheet configuration.

Both T17M and N15S mutants caused $\mathrm{RD}$, suggesting that glycosylation at N15 is essential for rhodopsin structure and/or function. Bovine N15Q rhodopsin, heterologously expressed in cultured cells, regenerates poorly with chromophore and exhibits decreased ability to activate transducin. In tunicamycin-treated cultured cells, nonglycosylated bovine rhodopsin expresses at normal levels but inefficiently activates transducin (Kaushal et al., 1994). Together, these results suggest that lack of glycosylation at N15 is specifically linked to a defect in signal transduction. However, rhodopsin mutants that are incapable of activating transducin (e.g., K296R) are not detrimental to rod cell viability (Cohen et al., 1993; Tam and Moritz, 2006, 2007; Tam et al., 2006). Thus, although reduced signal transduction attributable to lack of glycosylation may impact visual sensitivity, it is unlikely to be the cause of RD.

T4K, N15S, T17M, and N2S/N15S rhodopsins all caused significantly less $\mathrm{RD}$ when the animals were reared in the dark, indicating that the mutants in their inactive states were less toxic. According to crystal structures, no gross conformational changes are reported for the $\mathrm{N}$ terminus of opsin during chromophore binding or light activation (Salom et al., 2006; Park et al., 2008; Scheerer et al., 2008), suggesting that this domain remains relatively static. Thus, lack of glycosylation at N15 or amino acid substitutions surrounding N2 may allow movement of the N terminus during light activation, resulting in an unstable rhodopsin molecule. The carbohydrate at N15 may help maintain the structure of the $\mathrm{N}$ terminus of light-activated rhodopsin by participating in stabilizing molecular interactions or by steric hindrance. Disruption of the normal N-terminal structure could result in loss of disk structure, potentially attributable to poor packing efficiency of rhodopsin within the membrane or decreased ability to dimerize.

We cannot preclude the possibility that decreased toxicity in the dark may be attributable to a decrease in opsin synthesis during dark rearing such that the rate of mutant rhodopsin synthesis falls below the toxic level. In X. laevis, opsin synthesis is constant over the course of a normal $12 \mathrm{~h}$ light/dark cycle (Hollyfield et al., 1982; Green et al., 1995) but decreases during prolonged dark rearing (Hollyfield and Anderson, 1982). However, even after accounting for a $48 \%$ reduction in opsin synthesis (Hollyfield and Anderson, 1982), the maximum levels of mutant opsin produced in the dark still exceeded the toxic levels observed in cyclic light-reared animals by more than threefold. Nonetheless, additional studies will be necessary to demonstrate conclusively that it is the activated forms of the rhodopsin glycosylation mutants that are toxic.

Neither bovine glycosylation-deficient mutant (T4R or T17M) induced RD in transgenic $X$. laevis when expressed at levels comparable with the analogous human mutants, suggesting that the bovine rhodopsin mutants may be more stable. Initially, this may seem surprising given the degree of sequence conservation between bovine and human rhodopsins. However, even single amino acid substitutions can significantly increase protein stability (Matthews et al., 1987; Kallwass et al., 1992; Goward et al., 1994; Takeshita et al., 2003). Moreover, bovine rhodopsin is considerably more thermostable than rhodopsins from other species. The Arrhenius energy of activation for the thermal denaturation of bovine rhodopsin is approximately double that of frog rhodopsin and the temperature for half-denaturation is greater for bovine rhodopsin (60 vs $54.5^{\circ} \mathrm{C}$ ) (Hubbard, 1958). Using differential scanning calorimetry, it was determined that, although rat and frog rhodopsin are very similar in stability, bovine rhodopsin is significantly more thermally stable $(\Delta H=410$, 416 , and $630 \mathrm{~kJ} / \mathrm{mol}$, respectively; $T d=340,340$, and $347^{\circ} \mathrm{K}$, respectively) (Shnyrov and Berman, 1988). Bovine rhodopsin is more stable toward hydroxylamine bleaching than canine rhodopsin (Zhu et al., 2004), indicating that the chromophore pocket of canine rhodopsin is more accessible and perhaps adopts a less compact structure. Finally, there are multiple examples of bovine rhodopsin mutants that fold and bind chromophore in cultured cells, producing photo-labile pigments, whereas the corresponding human mutants do not (Nathans, 1990a,b; Sung et al., 1991; Min et al., 1993; Sung et al., 1993; Kaushal et al., 1994). Thus, greater stability of bovine rhodopsin may account for the different outcomes observed with the human and bovine glycosylation mutants in this study.

The results obtained from this study as well as from our previous work on $\mathrm{P} 23 \mathrm{H}$ rhodopsin (Tam and Moritz, 2007) support the hypothesis that the sector RP phenotype is attributable to light-exacerbated RD. However, P23H rhodopsin likely involves a disease mechanism associated with a biosynthetic defect because the mutant displays decreased ability to exit from the ER. Despite conferring a similar light-sensitive phenotype, human rhodopsin glycosylation mutants appear to cause RD via a different mechanism that does not originate from the ER. This is striking considering that all of these mutations are contained within the same relatively small region of rhodopsin. Thus, although protection from light exposure may benefit patients harboring any of these $\mathrm{N}$-terminal mutations, pharmaceutical interventions 
may require more specific targeting toward the initiating pathogenic steps in cell death.

\section{References}

Adamus G, Zam ZS, Arendt A, Palczewski K, McDowell JH, Hargrave PA (1991) Anti-rhodopsin monoclonal antibodies of defined specificity: characterization and application. Vision Res 31:17-31.

Bourne HR, Meng EC (2000) Structure. Rhodopsin sees the light. Science 289:733-734.

Cideciyan AV, Jacobson SG, Aleman TS, Gu D, Pearce-Kelling SE, Sumaroka A, Acland GM, Aguirre GD (2005) In vivo dynamics of retinal injury and repair in the rhodopsin mutant dog model of human retinitis pigmentosa. Proc Natl Acad Sci U S A 102:5233-5238.

Cohen GB, Yang T, Robinson PR, Oprian DD (1993) Constitutive activation of opsin: influence of charge at position 134 and size at position 296. Biochemistry 32:6111-6115.

Conover WJ (1999) Practical nonparametric statistics, Ed 3. New York: Wiley.

Duffin KL, Lange GW, Welply JK, Florman R, O’Brien PJ, Dell A, Reason AJ, Morris HR, Fliesler SJ (1993) Identification and oligosaccharide structure analysis of rhodopsin glycoforms containing galactose and sialic acid. Glycobiology 3:365-380.

Filipek S, Stenkamp RE, Teller DC, Palczewski K (2003) G-Protein coupled receptor rhodopsin: a prospectus. Annu Rev Physiol 65:851-879.

Fishman GA, Stone EM, Sheffield VC, Gilbert LD, Kimura AE (1992) Ocular findings associated with rhodopsin gene codon 17 and codon 182 transition mutations in dominant retinitis pigmentosa. Arch Ophthalmol 110:54-62.

Fliesler SJ, Basinger SF (1985) Tunicamycin blocks the incorporation of opsin into retinal rod outer segment membranes. Proc Natl Acad Sci U S A 82:1116-1120.

Fliesler SJ, Rayborn ME, Hollyfield JG (1985) Membrane morphogenesis in retinal rod outer segments: inhibition by tunicamycin. J Cell Biol 100:574-587.

Fujita S, Endo T, Ju J, Kean EL, Kobata A (1994) Structural studies of the N-linked sugar chains of human rhodopsin. Glycobiology 4:633-640.

Goward CR, Miller J, Nicholls DJ, Irons LI, Scawen MD, O'Brien R, Chowdhry BZ (1994) A single amino acid mutation enhances the thermal stability of Escherichia coli malate dehydrogenase. Eur J Biochem 224:249-255.

Green CB, Cahill GM, Besharse JC (1995) Regulation of tryptophan hydroxylase expression by a retinal circadian oscillator in vitro. Brain Res 677:283-290.

Hargrave PA (1977) The amino-terminal tryptic peptide of bovine rhodopsin. A glycopeptide containing two sites of oligosaccharide attachment. Biochim Biophys Acta 492:83-94.

Hicks D, Molday RS (1986) Differential immunogold-dextran labeling of bovine and frog rod and cone cells using monoclonal antibodies against bovine rhodopsin. Exp Eye Res 42:55-71.

Hollyfield JG, Anderson RE (1982) Retinal protein synthesis in relationship to environmental lighting. Invest Ophthalmol Vis Sci 23:631-639.

Hollyfield JG, Rayborn ME, Verner GE, Maude MB, Anderson RE (1982) Membrane addition to rod photoreceptor outer segments: light stimulates membrane assembly in the absence of increased membrane biosynthesis. Invest Ophthalmol Vis Sci 22:417-427.

Hubbard R (1958) The thermal stability of rhodopsin and opsin. J Gen Physiol 42:259-280.

Kallwass HK, Surewicz WK, Parris W, Macfarlane EL, Luyten MA, Kay CM, Gold M, Jones JB (1992) Single amino acid substitutions can further increase the stability of a thermophilic L-lactate dehydrogenase. Protein Eng 5:769-774.

Kaushal S, Khorana HG (1994) Structure and function in rhodopsin. 7. Point mutations associated with autosomal dominant retinitis pigmentosa. Biochemistry 33:6121-6128.

Kaushal S, Ridge KD, Khorana HG (1994) Structure and function in rhodopsin: the role of asparagine-linked glycosylation. Proc Natl Acad Sci U S A 91:4024-4028.

Kijas JW, Cideciyan AV, Aleman TS, Pianta MJ, Pearce-Kelling SE, Miller BJ, Jacobson SG, Aguirre GD, Acland GM (2002) Naturally occurring rhodopsin mutation in the dog causes retinal dysfunction and degeneration mimicking human dominant retinitis pigmentosa. Proc Natl Acad Sci U S A 99:6328-6333.
Kroll KL, Amaya E (1996) Transgenic Xenopus embryos from sperm nuclear transplantations reveal FGF signaling requirements during gastrulation. Development 122:3173-3183.

Li T, Sandberg MA, Pawlyk BS, Rosner B, Hayes KC, Dryja TP, Berson EL (1998) Effect of vitamin A supplementation on rhodopsin mutants threonine-17 $\rightarrow$ methionine and proline- $347 \rightarrow$ serine in transgenic mice and in cell cultures. Proc Natl Acad Sci U S A 95:11933-11938.

Li ZY, Jacobson SG, Milam AH (1994) Autosomal dominant retinitis pigmentosa caused by the threonine-17-methionine rhodopsin mutation: retinal histopathology and immunocytochemistry. Exp Eye Res 58:397-408.

Luo W, Marsh-Armstrong N, Rattner A, Nathans J (2004) An outer segment localization signal at the $\mathrm{C}$ terminus of the photoreceptor-specific retinol dehydrogenase. J Neurosci 24:2623-2632.

MacKenzie D, Arendt A, Hargrave P, McDowell JH, Molday RS (1984) Localization of binding sites for carboxyl terminal specific anti-rhodopsin monoclonal antibodies using synthetic peptides. Biochemistry 23:65446549.

Matthews BW, Nicholson H, Becktel WJ (1987) Enhanced protein thermostability from site-directed mutations that decrease the entropy of unfolding. Proc Natl Acad Sci U S A 84:6663-6667.

Maw MA, Corbeil D, Koch J, Hellwig A, Wilson-Wheeler JC, Bridges RJ, Kumaramanickavel G, John S, Nancarrow D, Röper K, Weigmann A, Huttner WB, Denton MJ (2000) A frameshift mutation in prominin (mouse)-like 1 causes human retinal degeneration. Hum Mol Genet 9:27-34.

Min KC, Zvyaga TA, Cypess AM, Sakmar TP (1993) Characterization of mutant rhodopsins responsible for autosomal dominant retinitis pigmentosa. Mutations on the cytoplasmic surface affect transducin activation. J Biol Chem 268:9400-9404.

Moritz OL, Tam BM, Knox BE, Papermaster DS (1999) Fluorescent photoreceptors of transgenic Xenopus laevis imaged in vivo by two microscopy techniques. Invest Ophthalmol Vis Sci 40:3276-3280.

Moritz OL, Biddle KE, Tam BM (2002) Selection of transgenic Xenopus laevis using antibiotic resistance. Transgenic Res 11:315-319.

Nathans J (1990a) Determinants of visual pigment absorbance: role of charged amino acids in the putative transmembrane segments. Biochemistry 29:937-942.

Nathans J (1990b) Determinants of visual pigment absorbance: identification of the retinylidene Schiff s base counterion in bovine rhodopsin. Biochemistry 29:9746-9752.

Nelson RM, Long GL (1989) A general method of site-specific mutagenesis using a modification of the Thermus aquaticus polymerase chain reaction. Anal Biochem 180:147-151.

Palczewski K, Kumasaka T, Hori T, Behnke CA, Motoshima H, Fox BA, Le Trong I, Teller DC, Okada T, Stenkamp RE, Yamamoto M, Miyano M (2000) Crystal structure of rhodopsin: a G protein-coupled receptor. Science 289:739-745.

Park JH, Scheerer P, Hofmann KP, Choe HW, Ernst OP (2008) Crystal structure of the ligand-free G-protein-coupled receptor opsin. Nature 454:183-187.

Salom D, Lodowski DT, Stenkamp RE, Le Trong I, Golczak M, Jastrzebska B, Harris T, Ballesteros JA, Palczewski K (2006) Crystal structure of a photoactivated deprotonated intermediate of rhodopsin. Proc Natl Acad Sci U S A 103:16123-16128.

Scheerer P, Park JH, Hildebrand PW, Kim YJ, Krauss N, Choe HW, Hofmann KP, Ernst OP (2008) Crystal structure of opsin in its G-proteininteracting conformation. Nature 455:497-502.

Shnyrov VL, Berman AL (1988) Calorimetric study of thermal denaturation of vertebrate visual pigments. Biomed Biochim Acta 47:355-362.

Sullivan LJ, Makris GS, Dickinson P, Mulhall LE, Forrest S, Cotton RG, Loughnan MS (1993) A new codon 15 rhodopsin gene mutation in autosomal dominant retinitis pigmentosa is associated with sectorial disease. Arch Ophthalmol 111:1512-1517.

Sung CH, Schneider BG, Agarwal N, Papermaster DS, Nathans J (1991) Functional heterogeneity of mutant rhodopsins responsible for autosomal dominant retinitis pigmentosa. Proc Natl Acad Sci U S A 88:8840-8844.

Sung CH, Davenport CM, Nathans J (1993) Rhodopsin mutations responsible for autosomal dominant retinitis pigmentosa. Clustering of functional classes along the polypeptide chain. J Biol Chem 268:26645-26649.

Takeshita H, Yasuda T, Nakajima T, Mogi K, Kaneko Y, Iida R, Kishi K 
(2003) A single amino acid substitution of Leu130Ile in snake DNases I contributes to the acquisition of thermal stability. Eur J Biochem 270:307-314.

Tam BM, Moritz OL (2006) Characterization of rhodopsin P23H-induced retinal degeneration in a Xenopus laevis model of retinitis pigmentosa. Invest Ophthalmol Vis Sci 47:3234-3241.

Tam BM, Moritz OL (2007) Dark rearing rescues P23H rhodopsin-induced retinal degeneration in a transgenic Xenopus laevis model of retinitis pigmentosa: a chromophore-dependent mechanism characterized by production of N-terminally truncated mutant rhodopsin. J Neurosci 27: 9043-9053.

Tam BM, Xie G, Oprian DD, Moritz OL (2006) Mislocalized rhodopsin does not require activation to cause retinal degeneration and neurite outgrowth in Xenopus laevis. J Neurosci 26:203-209.

van den Born LI, van Schooneveld MJ, de Jong LA, Riemslag FC, de Jong PT, Gal A, Bleeker-Wagemakers EM (1994) Thr4Lys rhodopsin mutation is associated with autosomal dominant retinitis pigmentosa of the cone-rod type in a small Dutch family. Ophthalmic Genet 15:51-60.

White DA, Fritz JJ, Hauswirth WW, Kaushal S, Lewin AS (2007) Increased sensitivity to light-induced damage in a mouse model of au- tosomal dominant retinal disease. Invest Ophthalmol Vis Sci 48: 1942-1951.

Williams RW, Chang A, Juretić D, Loughran S (1987) Secondary structure predictions and medium range interactions. Biochim Biophys Acta 916:200-204.

Wiseman RL, Powers ET, Buxbaum JN, Kelly JW, Balch WE (2007) An adaptable standard for protein export from the endoplasmic reticulum. Cell 131:809-821.

Yang Z, Chen Y, Lillo C, Chien J, Yu Z, Michaelides M, Klein M, Howes KA, Li Y, Kaminoh Y, Chen H, Zhao C, Chen Y, Al-Sheikh YT, Karan G, Corbeil D, Escher P, Kamaya S, Li C, Johnson S, Frederick JM, Zhao Y, Wang C, Cameron DJ, Huttner WB, Schorderet DF, Munier FL, Moore AT, Birch DG, Baehr W, Hunt DM, Williams DS, Zhang K (2008) Mutant prominin 1 found in patients with macular degeneration disrupts photoreceptor disk morphogenesis in mice. J Clin Invest 118:2908-2916.

Zhu L, Jang GF, Jastrzebska B, Filipek S, Pearce-Kelling SE, Aguirre GD, Stenkamp RE, Acland GM, Palczewski K (2004) A naturally occurring mutation of the opsin gene (T4R) in dogs affects glycosylation and stability of the G protein-coupled receptor. J Biol Chem 279:5382853839. 